\section{Art in Hospital}

\section{Zusammenfassung}

Seit April 2006 werden in den Räumlichkeiten der Station und Ambulanz der Hautklinik und des Immunologischen Zentrums des Städtischen Klinikums Dessau Fotografien von Mitarbeitern ausgestellt. Dabei sollen die Bilder eine angenehme Atmosphäre schaffen, den gedanklichen Austausch untereinander fördern, die Phantasie anregen und damit auch die Genesung der Patienten unterstützen. Das Krankenhaus mit Patienten aller sozialer Schichten und Altersgruppen ist ein Teil der Gesellschaft. Da ist es nur natürlich, auch dort Impulse zu vermitteln, die auch unserem normalen Alltagsleben Sinn und Gehalt geben: Kommunikation und Geborgenheit, Begegnung mit der Kunst.
Abstract

Since April 2006 photographs of employees are exhibited at the ward and the outpatient division of the Departments of Dermatology and Immunology, Dessau Medical Center. The photograph exhibition aims to create a pleasant atmosphere, promote the intellectual exchange, activate the imagination and thus also support the recovery of the patients. The Medical Center treating patients of all social levels and age groups is part of the society. It is, therefore, comprehensive that such impulses are communicated, which are also perceived in normal everyday life: feelings of communication and security; meeting with the art.
Seit Mitte April 2006 werden in den Räumlichkeiten der Station und Ambulanz der Hautklinik und des Immunologischen Zentrums des Städtischen Klinikums Dessau Fotografien mit Motiven aus der Region Dessau und einiger europäischer Länder gezeigt (Abb.1-9). Die Bilder stammen aus den als Hobby entstandenen Sammlungen von Herrn Prof. Dr. Christos C. Zouboulis (Ambulanz) und Herrn Dr. Dietrich Trebing (Station).

Bei der Motivwahl ging es weniger um Sehenswürdigkeiten als solche, sondern vielmehr um die Widerspiegelung bestimmter Stimmungen und besonderer Augenblicke an vielfältigen Schauplätzen von Natur, Landschaft, Parks und Regionen [1]. Dabei sollen die Bilder nicht nur bloße Dekoration sein, sondern eine angenehme Atmosphäre schaffen, den gedanklichen Austausch untereinander fördern, die Phantasie anregen und damit auch die
Genesung unterstützen [2]. Das Krankenhaus mit Patienten aller sozialer Schichten und Altersgruppen ist ein Teil der Gesellschaft. Da ist es nur natürlich, auch dort Impulse zu vermitteln, die auch unserem normalen Alltagsleben Sinn und Gehalt geben: Kommunikation und Geborgenheit, Begegnung mit der Kunst [1].

„Ein gutes Foto ist ein Foto, auf das man länger als eine Sekunde schaut.“ (Henri Cartier-Bresson, französischer Fotograf, Regisseur, Schauspieler, 1908-2004).

Die Resonanz bei Patienten, Besuchern und Mitarbeitern war bereits in den ersten Ausstellungstagen unerwartet groß und durchweg positiv. In einer kleinen Bewertungsaktion können die Betrachter ihre Eindrücke wiedergeben, die Teilnahme mit 


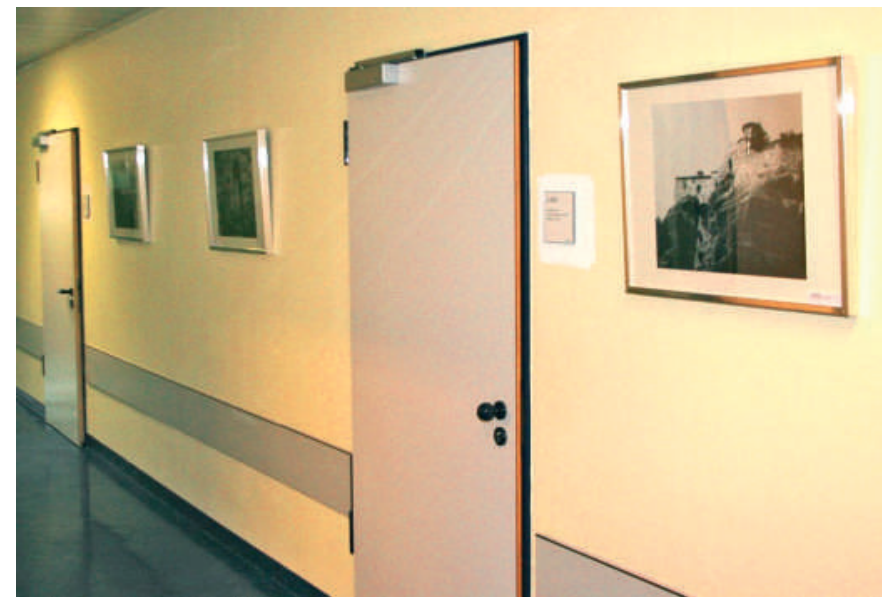

Abb. 1 Flur der Hautambulanz.
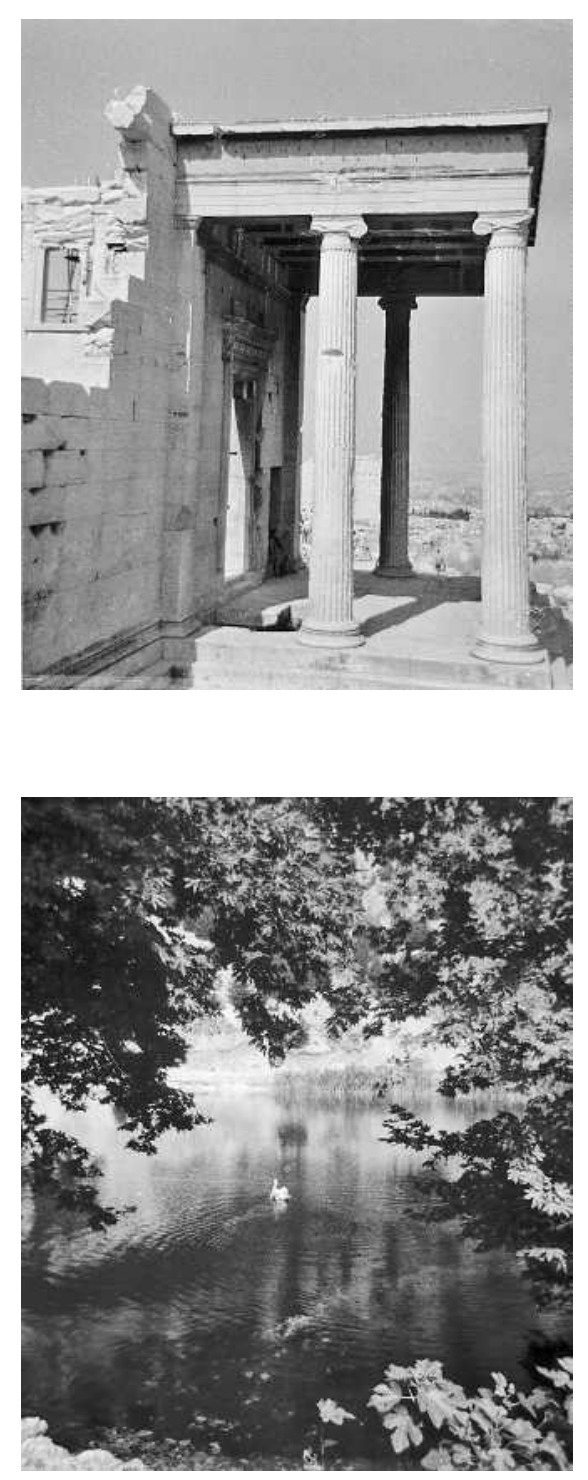

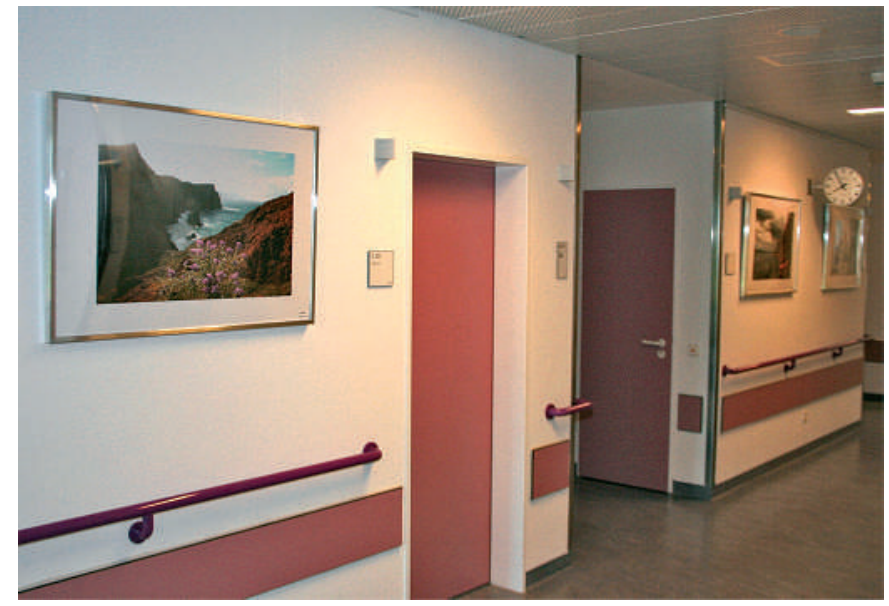

Abb. 4 Flur der Hautstation.

Abb. 2 „Licht/Schatten", Erechthion (Akropolis, Athen, GR).

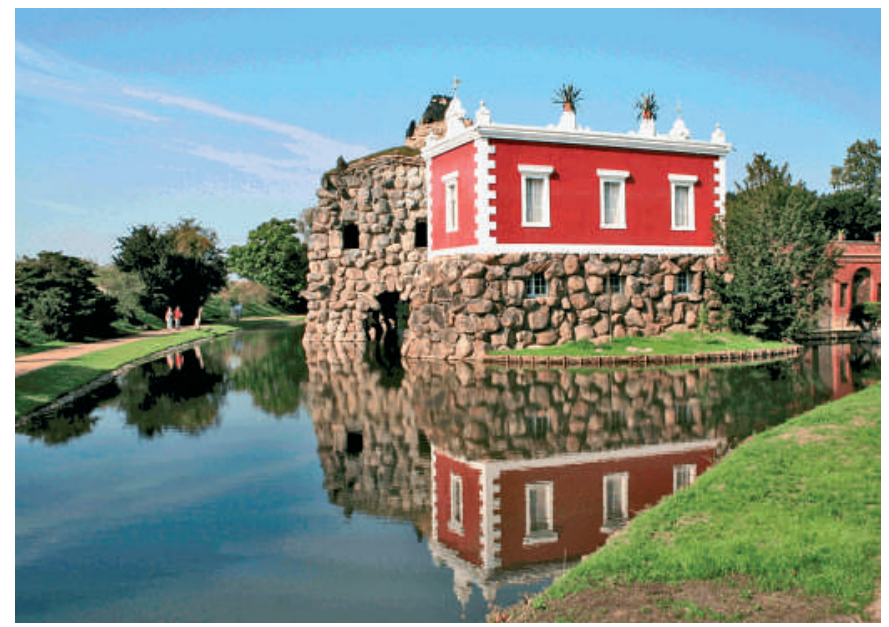

Abb. 5 Villa Hamilton (Wörlitzer Park, D).

Abb. 3 „Schwanensee", Louros Fluss (Hepirus, GR).

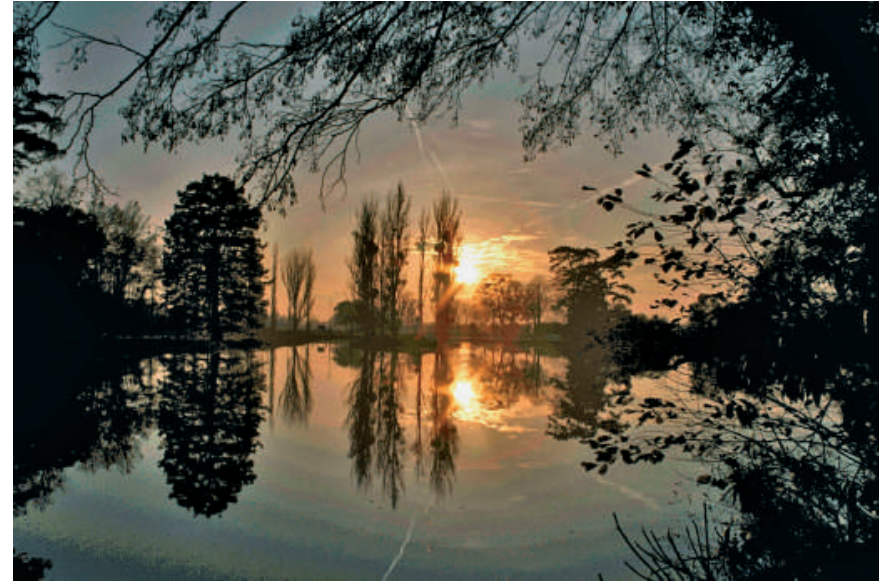

Abb. 6 Rousseau-Insel (Wörlitzer Park, D). 


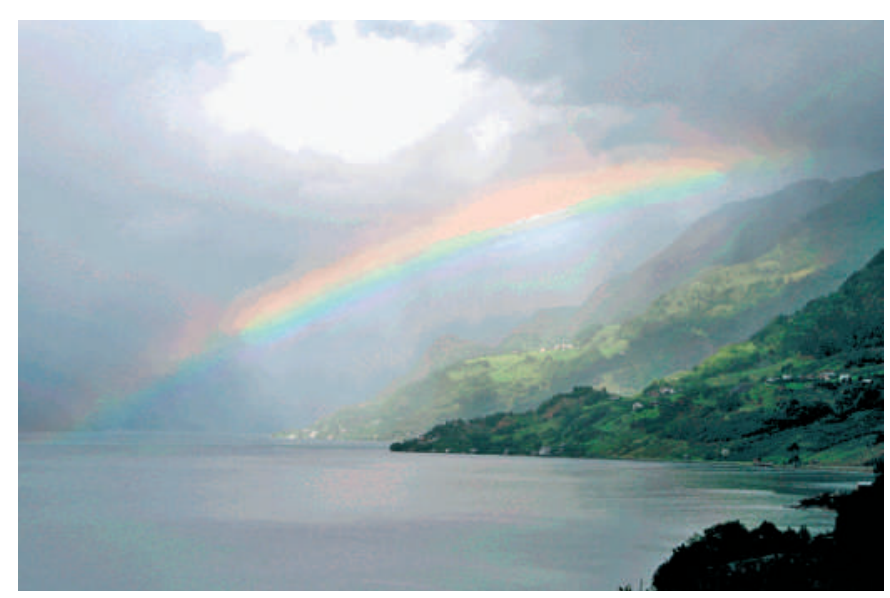

Abb. 7 Gewitterstimmung (Hardangerfjord, N).

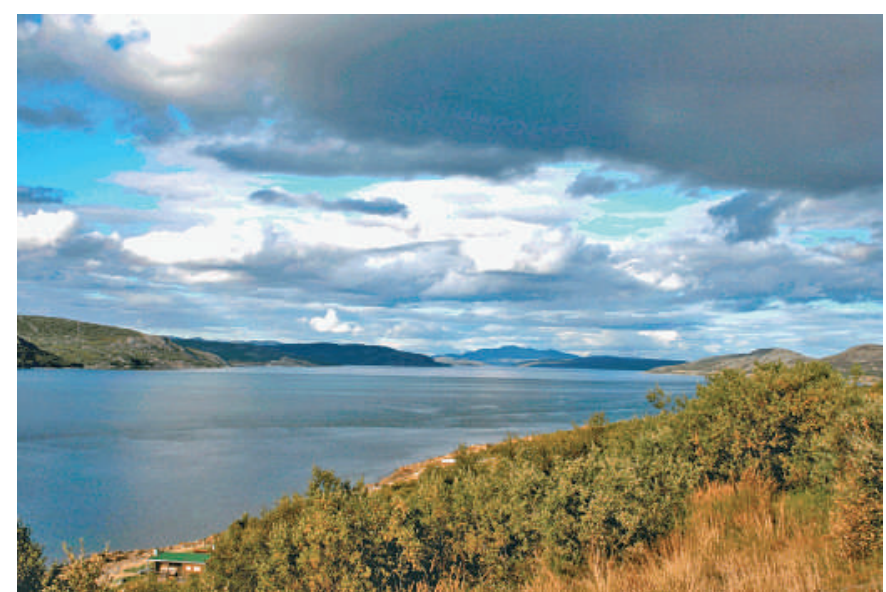

Abb. 8 Fjordlandschaft (Finnmark, N).

über 50 abgegebenen Stimmen innerhalb der ersten 4 Wochen reflektiert das deutliche Interesse und bestätigt uns in dem Vorhaben. Die „außermedizinische“ Abwechslung wird vor allem von den stationären Patienten dankbar und mit viel Freude angenommen und hilft vielleicht auch ein wenig, sich in die sonst etwas nüchterne, ungewohnte Umgebung einzufügen. Auch den Mitarbeitern soll die Ausstellung natürlich eine kleine Aufmunterung sein.

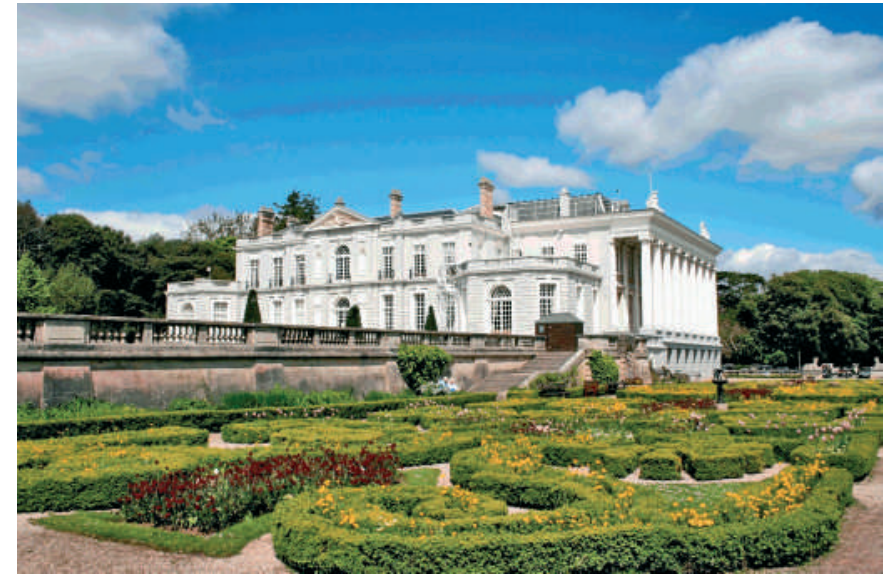

Abb. 9 Oldway-Mansion (Paignton, GB).

Die fakultativen Bewertungsbögen werden zum Teil akribisch ausgefüllt, neue Bildtitel entworfen und Kinder bitten Eltern oder Mitpatienten um Hilfestellung beim Schreiben. Übereinstimmend geben viele an, gar keine „Rangfolge“ treffen zu können, da ihnen alle Bilder sehr gut gefallen. Eine Besucherin schrieb als Kommentar: „Die Entscheidung fällt sehr schwer. Tolle Motive, man bekommt Fernweh. Viel besser als Kunstdrucke, die überall zu finden sind.“

Bei dem Vorhaben, finanziell unterstützt durch die Verwaltung des Klinikums, planen wir in Zukunft, die Ausstellung in bestimmten Abständen mit wechselnden Motiven unterschiedlicher Künstler zu versehen.

Die an Schienen befestigten Wechselrahmen gestatten künftig auch die Präsentation von Gemälden, Handarbeiten und anderen Kunstwerken.

\section{Literatur}

${ }^{1}$ Manion SM, Nowicki C. St. John's Regional Health Center. Photographs with a personal touch create pleasant surroundings. Health Prog 1992; 73: $82-83$

${ }^{2}$ Folk art reduces hospital stress. Hosp Physician 1990; 26: 67-69 